\title{
Atendimento odontológico domiciliar ao idoso e a necessidade de tratamento endodôntico
}

\author{
The home dental care to elderly and the need for endodontic treatment
}

Roberta Cordeiro Fuster Vieira

Pós-graduação em Endodontia pela UNIG

Cirurgiã-Dentista do Serviço Integrado de Assistência Domiciliar do Hospital Naval Marcílio Dias (HNMD)

\section{Carlos Alberto dos Santos}

Pós-graduação em Odontopediatria pela Odontoclínica Central da Marinha (OCM)

Cirurgião-Dentista do Serviço Integrado de Assistência Domiciliar do HNMD

\section{Narahyana Bom de Araujo}

Mestre em Saúde Mental pela UFRJ

Psicóloga do Serviço Integrado de Assistência Domiciliar do HNMD

\section{Raphael Cordeiro da Cruz}

Pós-graduação em Geriatria e Gerontologia pela Unesa

Geriatra do Serviço Integrado de Assistência Domiciliar do HNMD

\section{Edir Gomes de Azevedo}

Técnico de enfermagem do Serviço Integrado de Assistência Domiciliar do HNMD

Gisele Mendes de Souza e Mello

Pós-graduação em Geriatria pela Sociedade Brasileira de Geriatria e Gerontologia e Associação Médica Brasileira

Chefe da Clínica de Geriatra e Encarregada do Serviço Integrado de Assistência Domiciliar do HNMD

\section{Resumo}

$\mathrm{O}$ objetivo deste trabalho foi realizar um estudo transversal dos pacientes atendidos pelo Serviço Integrado de Atendimento Domiciliar do Hospital Naval Marcílio Dias e relatar a necessidade que eles apresentam de tratamento endodôntico. Duzentos e setenta e cinco pacientes foram avaliados através de exame clínico (visual-tátil), sendo avaliada a presença ou ausência de dentes e a necessidade de intervenção. Assim, os pacientes foram divididos em três grupos: grupo indicado para extração, outro para Endodontia e restauração e um terceiro para extração e Endodontia em elementos dentários diferentes. Os dados da avaliação odontológica foram levantados e os percentuais avaliados.

Palavras-chave: idoso; Endodontia; atendimento domiciliar.

\section{Abstract}

The aim of this study was a cross-sectional study of patients seen by the Integrated Home Care Service, linked to the Hospital Naval Marcilio Dias and the need they have of endodontic treatment. Were evaluated by clinical examination (visual-tactile) 275 patients. These patients were divided into three groups, extraction was indicated, designated endodontics and subsequent restoration and indicated extraction and endodontics in different dental elements. The dental evaluation data were collected and evaluated percentage.

Keywords: elderly; Endodontics; home care.

\section{Introdução}

A população mundial está envelhecendo, principalmente nos países em desenvolvimento como o Brasil. A faixa etária a partir de 60 anos é a que mais aumenta em termos proporcionais. Projeções da Organização Mundial de Saúde (OMS) evidenciam que a população de idosos brasileiros, no período de 1950 a 2025, deverá ter aumentado em quase 15 vezes, enquanto o restante da população terá aumentado em cinco vezes. Frente a essa realidade, diversos setores deverão passar por reestruturações para assegurar a inclusão, na família e na sociedade de um modo geral, de um contingente cada vez maior de idosos $(1,2,3)$.

Diante deste cenário, a assistência domiciliar emerge como uma alternativa tanto para a melhora na utilização dos recursos em saúde, quanto para a promoção e manutenção da saúde, a melhora da independência, a diminuição dos efeitos da incapacidade funcional ou da atividade da doença. Podemos considerar a assistência domiciliar como uma modalidade de assistência em que um conjunto de atividades de caráter ambulatorial são programadas e continuamente desenvolvidas no domicilio do paciente $(4,5)$.

Visando promover a atenção qualificada ao idoso, em cumprimento à Política Nacional do Idoso e ao Estatuto do Idoso, a Marinha do Brasil (MB) criou, em setembro de 2009, o Serviço Integrado de Atendimento Domiciliar (SIAD). O serviço é composto por uma equipe de saúde multidisciplinar, vinculada à clínica de geriatria do Hospital Naval Marcílio Dias (HNMD), responsável pela prestação da assistência domiciliar na área do Rio de Janeiro, destinada aos pacientes que, por limitação funcional ou problema de saúde, não podem manter acompanhamento nos ambulatórios da MB. Atualmente, a equipe multidisciplinar do SIAD é composta por médicos, enfermeiro e técnicos de enfermagem, assistente social, dentista e psicóloga. Esta equipe é responsável pelo planejamento do plano terapêutico individualizado, assim como pela supervisão do atendimento domiciliar prestado por empresas terceirizadas e credenciadas $(6,7,8)$.

Dentro do panorama apresentado a odontologia domiciliar pode ser considerada como mais uma área de atuação odontológica a ser realizada pelo cirurgião-dentista, com ênfase multidisciplinar, em que se avalia o paciente como um todo, contribuindo assim para a promoção da qualidade de vida $\mathrm{e}$ independência funcional para as pessoas assistidas $(1,4,2)$.

No atendimento odontológico domiciliar da MB que compõe o SIAD, os dentistas realizam procedimentos de prevenção, promoção de saúde oral, eliminação de foco de inflamação e/ou infecção e sintomatologia dolorosa. Após avaliação inicial, caso o paciente tenha necessidade de intervenção, pode ser agendado para realizar um procedimento simples no domicílio ou ser encaminhado para a clínica de Odontogeriatria da Odontoclínica Central da Marinha (OCM) ou ainda ser encaminhado para procedimento na Clínica de Bucomaxilofacial do HNMD, conforme demonstra o Fluxograma do SIAD abaixo (figura 1). 
Figura 1. Fluxograma do SIAD

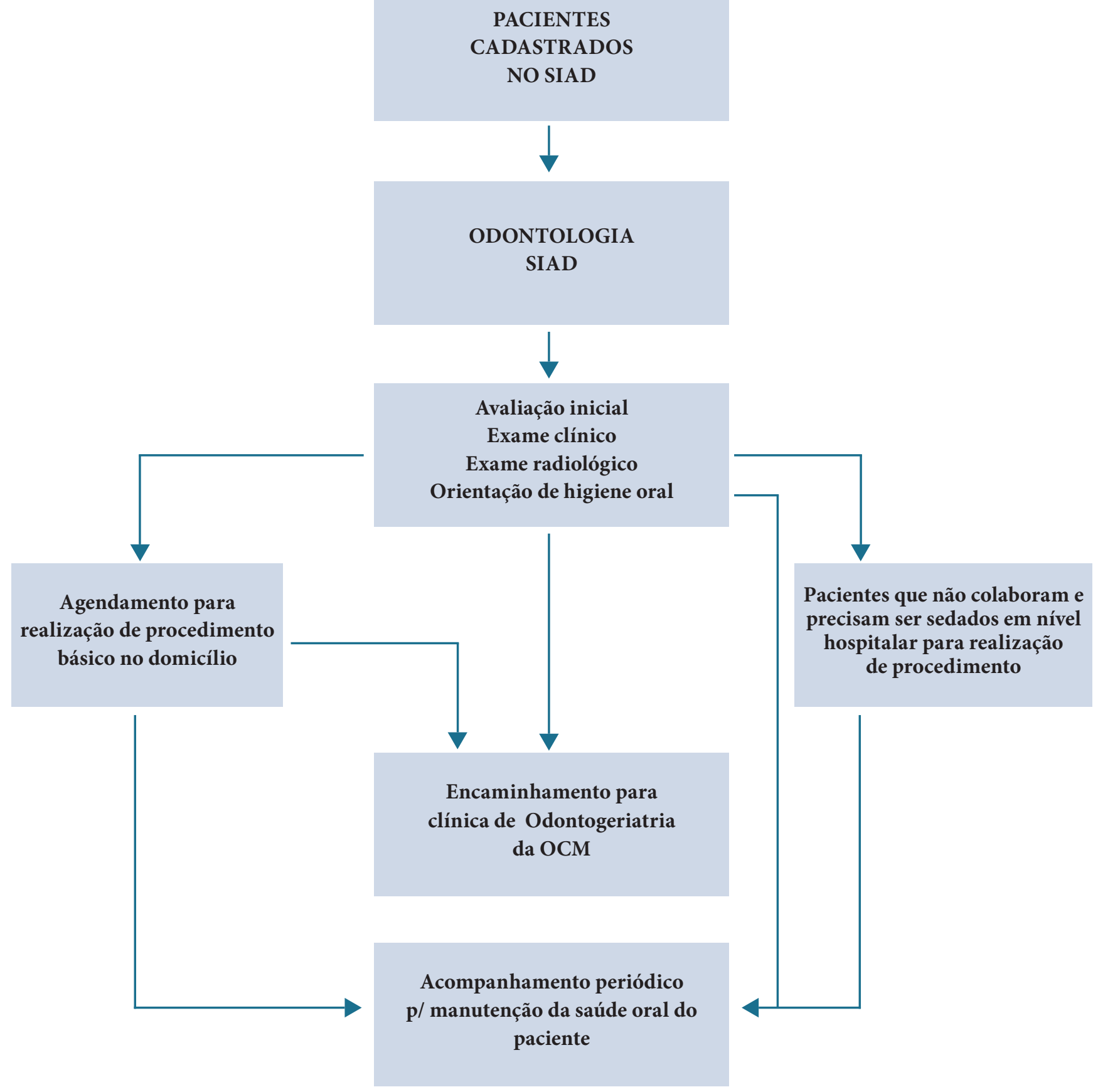

Todo esse processo de avaliação e encaminhamento para intervenção visa tratar e reabilitar, sempre que possível, os dentes destes idosos, evitando, assim, realizar extrações desnecessárias. A opção por se manter os dentes naturais saudáveis e funcionais é fundamental para a saúde do idoso, influenciando na mastigação, na percepção do sabor dos alimentos, na fala e na estética. Por outro lado, a extração dos dentes pode provocar alterações nutricionais e comprometimento da saúde geral do idoso $(9,10)$. Dessa forma, há uma crescente necessidade da atuação do especialista em Endodontia. A Endodontia é a especialidade da Odontologia responsável pelo estudo de todo o sistema de canais radiculares e dos tecidos periapicais, bem como das doenças que os afligem. Em casos de alterações por fraturas dentárias, trauma dentário, trauma ortodôntico, lesões endoperiodontais, necessidades protéticas e outras patologias endodônticas, o tratamento endodôntico está indicado. O objetivo fim da Endodontia é a manutenção do dente na cavidade bucal e a saúde dos tecidos periapicais (11).

Nesse sentido, o objetivo deste trabalho foi avaliar odontologicamente os idosos cadastrados no SIAD de janeiro até julho de 2015, verificando a presença/ausência de dentes, avaliando a indicação para a extração ou para tratamento en- 
dodôntico/reabilitador. Destacando que o tratamento endodôntico/reabilitador evitaria o aumento do edentulismo e suas consequências, conforme supracitado.

\section{Material e Métodos}

O presente estudo foi aprovado pelo comitê de Ética e Pesquisa do Hospital Naval Marcílio Dias.

Essa é uma pesquisa de base domiciliar e transversal. Foram selecionados pacientes com idade igual ou superior a 60 anos, atendidos pelo SIAD entre os meses de janeiro até julho de 2015 e avaliados pela equipe de Odontologia. De uma amostra total de 326 pacientes atendidos pelo SIAD, foram inseridos na amostra final para este estudo 275 pacientes.

Para a avaliação odontológica foi realizado exame clínico (visual-tátil) com emprego de sonda exploradora de ponta arredondada, espelho bucal, cadeira comum e luz artificial com ou sem auxiliar de diagnóstico (escovação e secagem prévia ao exame). Inicialmente, foi avaliada a presença ou ausência de dentes. Quando os dentes estavam presentes, foi então avaliada a necessidade ou não de intervenção. Após essa avaliação, os pacientes foram divididos em três grupos, um primeiro grupo indicado para realizar extração, um segundo grupo indicado para realizar Endodontia e, posteriormente, restauração e um terceiro grupo indicado para fazer extração e Endodontia em elementos dentários diferentes. Os dados da avaliação odontológica foram levantados e os percentuais avaliados. As informações sociodemográficas e clínicas foram levantadas através dos dados inseridos no prontuário eletrônico dos pacientes.

\section{Resultados}

Foram avaliados 275 pacientes, com média etária de 78,62 (DP: $\pm 8,73$ ), a maioria dos pacientes, $57,8 \%$ eram do sexo feminino, 35,3\% eram militares da reserva/reformado, 33,1\% dependentes e 30,2\% eram pensionistas. Em relação ao diagnóstico principal, 111 pacientes tinham diagnóstico de Síndrome demencial, 93 pacientes sofreram AVC e 37 possuíam diagnóstico de Doença de Parkinson. Quanto às comorbidades, 105 pacientes apresentam hipertensão arterial e 46 pacientes Diabetes. Alguns pacientes apresentaram mais de um diagnóstico principal e mais de uma comorbidade. O quadro I apresenta os dados sociodemográficos e clínicos.

Quadro I. Dados sociodemográficos e clínicos

\begin{tabular}{|c|c|}
\hline Total: 275 & \\
\hline Idade & $78,62( \pm 8,73)$ \\
\hline \multicolumn{2}{|l|}{ Gênero } \\
\hline Feminino (\%) & $57,8 \%$ \\
\hline Masculino (\%) & $42,2 \%$ \\
\hline \multicolumn{2}{|l|}{ Posto/Graduação } \\
\hline Militar reserva/reformado & $35,3 \%$ \\
\hline Dependente & $33,1 \%$ \\
\hline Pensionista & $30,2 \%$ \\
\hline Funcionário Civil & $0,7 \%$ \\
\hline Ex-Combatente & $0,7 \%$ \\
\hline Diagnóstico Principal & $\mathbf{n}$ \\
\hline Síndrome Demencial & 111 \\
\hline Doença de Parkinson & 37 \\
\hline AVC & 93 \\
\hline Cardiopatia & 13 \\
\hline Fratura/lesão ortopédica & 16 \\
\hline Obesidade & 9 \\
\hline Principais comorbidades & $\mathbf{n}$ \\
\hline Hipertensão Arterial & 105 \\
\hline Diabetes & 46 \\
\hline Depressão & 9 \\
\hline Doença Psiquiátrica & 4 \\
\hline Câncer & 4 \\
\hline
\end{tabular}

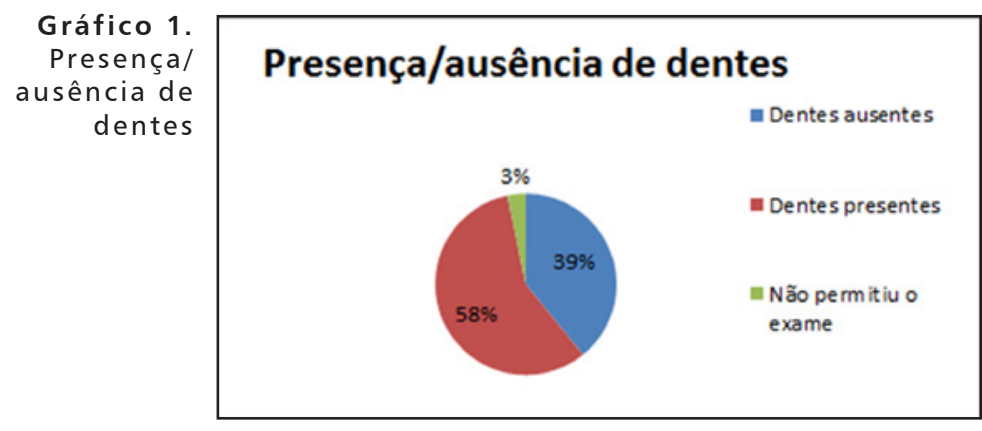

Gráfico 2 . Necessidade de intervenção

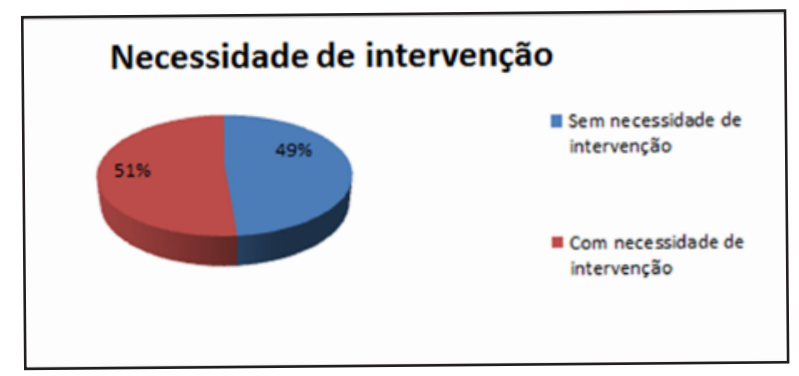

Gráfico 3. Indicação de intervenção

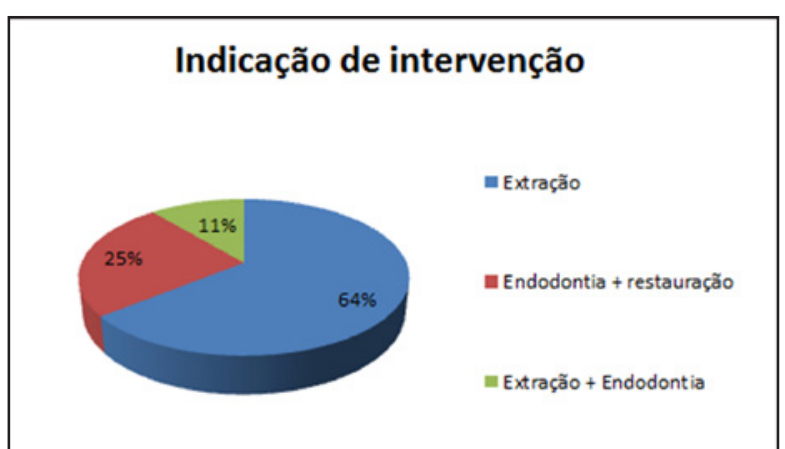


Dentre os 275 pacientes, a maioria, 58\% apresenta algum dente presente, conforme gráfico 1.

Em relação àqueles que tinham dentes presentes, o estudo evidenciou que $51 \%$ apresentavam necessidade de intervenção, como demonstrado no gráfico 2 .

Dentre aqueles pacientes que apresentaram necessidade de intervenção, 64\% tiveram indicação de extração, 25\% de Endodontia e posterior restauração e 11\% de extração e Endodontia em elementos dentários diferentes. Conforme gráfico 3.

\section{Discussão}

Esse estudo avaliou odontologicamente idosos cadastrados no SIAD de janeiro até julho de 2015, verificando a presença/ ausência de dentes, avaliando a indicação para a extração ou para tratamento endodôntico/reabilitador. Esses pacientes foram avaliados em suas residências através de exame clínico (visual-tátil) com emprego de sonda exploradora, espelho bucal, cadeira comum e luz artificial com ou sem auxiliar de diagnóstico.

Os resultados desse estudo mostram um índice alto de edentulismo (39\%), resultado próximo ao nosso foi verificado em estudo anterior também com idosos do Estado do Rio de Janeiro, em que foram encontrados $44 \%$ de ausência de dentes na amostra estudada. Dos $58 \%$ dos pacientes que possuíam dentes, $51 \%$ apresentavam necessidade de intervenção. Esses pacientes foram separados em três grupos de acordo com a intervenção indicada, a maior parte deles $64 \%$ foi indicada à extração, em $25 \%$ indicou-se Endodontia e posterior restauração e em $11 \%$ foram indicadas extração e Endodontia em elementos dentários diferentes. Dessa forma, cerca de 36\% dos pacientes apresentavam indicação para Endodontia. Verifica-se, assim, um aumento da demanda de tratamento endodôntico para os idosos, anteriormente à extração consistia a principal ou até mesmo única alternativa. Esse incremento na demanda por tratamento endodôntico deve-se também ao aumento da expectativa de vida, mas também há uma maior aceitação do tratamento endodôntico na terceira idade $(9,11)$. Estudos demonstram que conservar em média 20 dentes remanescentes nos idosos é garantia de melhor saúde geral e maior sobrevida $(12,13)$, além de ser fundamental para a mastigação, fala e percepção do sabor (9).

A modalidade de assistência domiciliar torna-se um facilitador, já que o endodontista atua na residência do paciente, avaliando clinicamente o idoso e encaminhando para a intervenção quando indicada. Pequenas intervenções podem ser realizadas no próprio domicílio como a prevenção e promoção de saúde oral, a eliminação de foco de inflamação e/ou infecção e da sintomatologia dolorosa. Aqueles pacientes que necessitam de uma intervenção que não pode ser realizada em sua residência são encaminhados para a clínica de Odontogeriatria da OCM ou a clínica de Bucomaxilofacial do HNMD.

\section{Conclusão}

A atenção ao idoso e o cuidado para manutenção de sua saúde são fundamentais para a qualidade de vida. A saúde bucal tem um papel relevante nesse contexto, visto que uma saúde bucal comprometida pode afetar o nível nutricional, o bem-estar físico e mental e diminuir o prazer de uma vida social mais ativa (14). Dessa forma, o tratamento odontológico é um dos aspectos a ser considerado na saúde geral do idoso, que por muitas vezes é pouco valorizado ou não se é dada a devida atenção.

Os resultados do presente estudo demonstram que a maior parte dos pacientes idosos avaliados apresenta elemento dentário na cavidade oral e com necessidade de intervenção. Dentre as vantagens de se manter os dentes naturais estão os benefícios para a mastigação, para a percepção do sabor dos alimentos, para a fala e para a estética $(9,10)$. Contudo, a decisão de extração ou não de um elemento dental comprometido endodonticamente em paciente idoso não deve ser baseada na idade do paciente, mas em uma avaliação de fatores gerais e locais, prós e contras para a preservação do dente (9), visando à saúde geral deste paciente. Dessa forma, a atuação do especialista em Endodontia, com experiência no atendimento geriátrico, é fundamental nesta avaliação e se torna cada vez mais necessária, visto que a demanda por tratamentos endodônticos para a terceira idade tem aumentado significativamente. 


\section{Referências ::}

1. Miranda AF, Montenegro FLB. O Cirurgião-dentista como parte integrante da equipe multidisciplinar direcionada à população idosa dependente no ambiente do domiciliar. Rev. paul. Odontol. 2009;31(3):15-9.

2. Marques GC, Montenegro FLB, Bezerra LF, et al. Odontologia domiciliar ao idoso frágil: a importância da Odontogeriatria. Revista portal de Divulgação, 42, Ano V. 2014. Disponível em www.portaldoenvelhecimento.org.br/revista

3. Instituto Brasileiro de Geografia e Estatística - IBGE. Projeção da População do Brasil por sexo e idade: 1980-2050. Rio de Janeiro, IBGE, 2008.

4. Rocha DA, Miranda AF. Atendimento odontológico domiciliar aos idosos: uma necessidade na prática multidisciplinar em saúde: revisão de literatura. Rev. bras. geriatr. gerontol. 2013;16(1):181-9.

5. Ministério da Saúde. Agência Nacional de Vigilância Sanitária. Resolução RDC nº11, de 26 de janeiro de 2006.

6. Brasil. Diretoria-Geral do Pessoal Da Marinha (DGPM) 401. Capítulo 17. Assistência Odontológica da Marinha do Brasil - Normas para Assistência Médico-Hospitalar. Rev.3 Mod.2, 2012.

7. LEI no 8.842 de 04 de janeiro de 1994. Política Nacional do Idoso.
8. LEI n ${ }^{\circ} 10.741$ de 01 de outubro de 2003. Estatuto do Idoso.

9. Santos MBF, Luthi LF, Zampieri MH, et al. Tratamento Endodôntico na terceira idade. RGO. 2003; 61:485-9.

10. Rocha NSM, Rocha AM, Aquino AMB, et al. Saúde bucal do idoso atendido na Policlínica Geral do Rio de Janeiro: uma análise radiográfica. Rev. Bras. de Odontol. 2012;69(2):238-41.

11. Souza KC, Veloso HHP, Queiroga AS. A perspectiva dos pacientes do serviço público de saúde de João Pessoa-PB frente ao tratamento endodôntico. Rev. Odontol. Bras. 2012;21(59):534-7.

12. Sheiham A, Steele JG, Marcenes W, et al. The relationship among dental status, nutrient intake anda nutricional status in older people. J. Dent. Res. 2001;80(2):408-13.

13. Shimazaki Y, Soh I, Saito T, et al. Influence of dentition satatus on physical disability, mental impairment and mortality in institutionalized elderly people. J. dent. 2001;80(1):340-5.

14. Rosa LB, Zuccolotto MCC, Bataglion C, et al. Odontogeriatria - a saúde bucal na terceira idade. RFO. 2008;13(2):82-6.

Recebido em: 10/08/2015 Aprovado em: 14/09/2015

Roberta Cordeiro Fuster Vieira

Avenida dos Flamboyants $n^{\circ} 100$, bloco 2 , apt ${ }^{\circ} 1102$

Rio de Janeiro/RJ, Brasil - CEP: 22776-070

E-mail: rcfuster@yahoo.com.br 